\title{
Proton Magnetic Resonance Spectroscopy Study on the Metabolism Changes of Cerebellum in Patients with Post-Stroke Depression
}

\author{
Lei Zhanga $^{\text {Ru-Bo Suib }}$
}

aSchool of Nursing, Liaoning Medical College, Jinzhou, Liaoning, b Department of Neurology, The First Affiliated Hospital of Jinzhou Medical University, Jinzhou, Liaoning, China

\section{Key Words}

Post-stroke depression • Cerebellum • Magnetic resonance spectroscopy • Depression severity

\begin{abstract}
Objective: To study the metabolic changes of cerebellum by proton magnetic resonance Spectroscopy ( $\left.{ }^{1} \mathrm{H}-\mathrm{MRS}\right)$ and discuss the relationships between the cerebellar changes and depression severity in patients with post-stroke depression. Methods and Results: Data of demographic characteristics, individual history and life style of all subjects were collected. 40 patients with stroke and 20 controls were enrolled. All groups received T1WI, T2WI, DWI and ${ }^{1} \mathrm{H}-\mathrm{MRS}$ examination. The cerebral infarction volume and the distribution and severity of leukoaraiosis were evaluated. The ratios of $\mathrm{NAA} / \mathrm{Cr}, \mathrm{Cho} / \mathrm{Cr}$ and $\mathrm{Cho} / \mathrm{NAA}$ in the cerebellum were calculated. There were no statistical significant difference in the $\mathrm{NAA} / \mathrm{Cr}$, $\mathrm{Cho} / \mathrm{Cr}$ and $\mathrm{Cho} /$ NAA ratios in bilateral cerebellum between CONT group and NORM group. The $\mathrm{Cho} / \mathrm{Cr}$ and Cho/NAA ratios in the cerebellum contralateral to the stroke region were higher in PSD group than those in NORM and CONT groups, and the Cho/Cr and Cho/NAA ratios in the cerebellum ipsilateral to the stroke region were similar with those in NORM and CONT groups. However, there were no statistical significant difference in the $\mathrm{NAA} / \mathrm{Cr}$ ratios in bilateral cerebellum among three groups. Conclusion: The result shows preliminarily that the cerebellum involves in the development of post-stroke depression.

\section{Introduction}

Post-stroke depression (PSD) refers a condition in which patients have 2-week or longer depressed mood or anhedonia after stroke attack [1]. PSD significantly affects the cognition, 
recovery and daily life of the stroke patients. It has been reported that the incidence of PSD was $28.1 \%$ in 2 weeks after stroke attack, 61\% within 1 year, even higher (79\%) in 2nd year following the stroke [2,3]. Nevertheless, the clinical efficacy of the current anti-depression agents is relatively limited [4]. Therefore, further investigating the pathogenesis of PSD and formulating the effective prevention measures are full of theoretic and realistic significance.

The pathogenesis of PSD remains largely elusive, the data from studies are contraversial. Traditional opinions suggest that stroke directly damage the neural circuit associated with depression such as the frontal lobes and temporal lobes, etc. However, a meta-analysis of 26 studies by Bhogal et al. showed that the relationship between PSD and infarction area was uncertain, and Altieri et al. found that the onset and severity of PSD were independent of the areas such as frontal lobes and temporal lobes [5, 6].

Cerebellum is not traditionally regarded as a part that is functionally associated with psychiatry or psychology disorders, while there are a growing evidence indicating that the cerebellum may be functionally involved in the control of cognition, behavior, and psychiatric illness [7-9]. As the most common psychiatric complication of stroke, PSD is a mental disorder that occurs after the attack of stroke, and usually refractory to conventional medical regimen. The mortality rates are relatively higher and recovery is delayed in the stroke patients with PSD than those without PSD.

${ }^{1} \mathrm{H}$-MRS is the only noninvasive molecular imaging method to inspect the change of local brain tissue metabolism at cell level in vivo, which makes it possible to determine the concentration of some neurotransmitters and the metabolism of some substances that could be functionally involved in the pathogenesis of the development of PSD [10]. The local blood flow and metabolism of brain will be altered when people have neural psychiatric activities, leading to a shift of ${ }^{1} \mathrm{H}-\mathrm{MRS}$ signal. In this way, MRS can be used to recognize the early PSD and achieve the purpose of early intervention and improvement of patients' limb and cognition function. Accumulative neuroimaging studies in recent years have showed that the mental disorders such as depression, infantile autism, etc. were associated with cerebellar lesions $[11,12]$.

Considering the observation that cerebellum is functionally related to the pathogenesis of mental disorders, and the fact that there is a similar pathogenesis shared between mental illnesses (especially for depression) and PSD, as well as the study showing that activated cerebella significantly alleviates symptom of PSD, we hypothesize that dysfunction of cerebellum may contribute to the development of PSD $[13,14]$. In this study, we used the clinical magnetic resonance spectrum imaging technique to quantitatively assess the cerebellar metabolic changes of PSD patients, and analyze its relationship with the severity of depression.

\section{Materials and Methods}

Study population selection and grouping

Post-stroke depression (PSD) group. The group included 20 inpatients from the Department of Neurology of First Affiliated Hospital of Liaoning Medical University between Mar.2014 to the Sep. 2015. The inclusive time was within 7 days after stroke attack. Inclusion criteria: (1) First-episode ischemic stroke attack limited to unilateral basal ganglia region (left or right), which was proved by Cranial MRI scan at the early onset of stroke and met the diagnostic criteria. (2) Depression was evaluated at day 14 after stroke attack: the results met the diagnostic criteria of depression from the Chinese Classification and Diagnostic Criteria of Mental Disorders (Version 3) (CCMD-3), meanwhile the Self-Rating Depression Scale (SDS) was used for depression screening. Those with standard scores $\geqq 50$ were included in the PSD group. Using Hamilton Anxiety Rating Scale for Depression (HAMD 24 items) for evaluating the severity of depression, those with scores $>7$ were definitely included in PSD group, and 8-17 scores were considered mild, 18-24 moderate, and $>24$ severe. (3) The patients must be accompanied by relatives in 2 weeks before and after the stoke attack. Exclusion criteria: (1) Age over 80 years old. (2) Past history of cerebrovascular diseases (cerebral infarction or cerebral hemorrhage) or a history of head injury or brain organic disease. (3) The criminal lesion suffered from now was not located in the basal ganglia. (4) Those with mental retardation, 


\section{Cellular Physiology Cell Physiol Biochem 2017;41:1393-1402

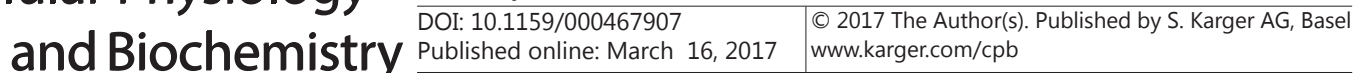

Zhang/Sui: Proton Magnetic Resonance Spectroscopy Study in PSD

severe vision and hearing impairment, aphasia and others affecting the inspection, or those who were not cooperate to complete the evaluation. (5) Those with a personal or family history of mental illness, such as depression and anxiety. (6) Those with a history of suspected alcohol and drug abuse.

Control (CONT) group. the group included 20 inpatients from the Department of Neurology of First Affiliated Hospital of Liaoning Medical University between Mar.2014 and Sep. 2015. The inclusive time was within 7 days after stroke attack. Inclusion criteria: (1) First-episode ischemic stroke attack limited to unilateral basal ganglia region (left or right), which was proved by Cranial MRI scan at the early onset of stroke and met the diagnostic criteria of acute ischemic stroke released by the Chinese Society of Neurology, CSN in 2014. (2) The patients must be accompanied by relatives in 2 weeks before and after the stoke attack. (3) The patients met the exclusion criteria for PSD group, but got a standard score $<50$ in SDS test and $\leqq 7$ in HAMD test in 14 days after disease onset, through which the depression was excluded.

Normal (NORM) group. 20 healthy volunteers possessing matched age and education degree with the subjects from PSD group. They all had no personal or family history of depression or anxiety, no history of brain trauma or cerebral organic disease, and all were right-handed. Depression was excluded through SDS scale and HAMD scale assessment.

This study was approved by the ethic committee of First Affiliated Hospital of Liaoning Medical University. All the subjects were fully informed and signed the content. There was no statistical deference in gender, age and education time between the CONT group, NORM group and PSD group.

\section{Collection of clinical data}

The following information of the subjects were recorded at the first visit: age, gender, family function, education level, occupation, economic condition, health insurance condition, past medical history (hypertension, diabetes, hyperlipidemia, cardiac disease) and previous life style (smoking, alcohol use, exercise, intake of fruit and vegetables). All information was provided by the patient and/or her/his relative. Cranial CT or MRI scan, echocardiography, ECG, carotid artery ultrasound and TCD were performed.

The patients had different classifications: physical work and mental work group based on their occupation; low and not low economic group according to monthly income per person in the family $\leq 1000$ yuan or $>1000$ yuan; and non-insured or insured persons [15]. Past medical history was recorded as 'Yes' or 'No'. Hypertension (SBP $\geq 140 \mathrm{mmHg}$ and/or DBP $\geq 90 \mathrm{mmHg}$ ), diabetes (random blood glucose $>$ $11.1 \mathrm{mmol} / \mathrm{L}$ ) and dyslipidemia (total cholesterol $>5.6 \mathrm{mmol} / \mathrm{L}$, total triglyceride $>1.7 \mathrm{mmol} / \mathrm{L}$, LDL-C $>$ $3.4 \mathrm{mmol} / \mathrm{L}$ or HDL-C $<0.9 \mathrm{mmol} / \mathrm{L}$ ) were defined as follows: this disease had been diagnosed by the doctor prior to the attack this time, or the corresponding medications had been administered, or it was newly diagnosed during this onset. The cardiac disease was defined as previously or newly diagnosed coronary disease, atrial fibrillation, valvulopathy, or history of pacemaker implantation or cardiac surgery [16]. Previous life style refers to the daily life style before the stroke attack, and smoking, alcohol use, exercise and intake of fruit and vegetables have been widely proved associated with stroke. Wherein, each factor may be healthy or unhealthy. Each unhealthy factor was counted as 1 , otherwise 0 , and the range of unhealthy factor number was 0 to 4 . Smoking was defined as smoking $>1$ cigarette per day for over 6 months. Excessive alcohol use is believed if consume $>50 \mathrm{ml}, 100 \mathrm{ml}$ and $300 \mathrm{ml}$ of white wine, red wine (or rice wine) and beer per day, respectively. Lack of exercise is considered in case of daily physical exercise time $<0.5 \mathrm{~h}$, and inadequate intake of fruits and vegetables was defined as daily intake < 450g [17].

\section{Methods}

Research tool. All the evaluation was done by the same clinically experienced and trained neurological doctor in a double-blind manner.

(1) Evaluation of family function satisfaction: the family APGAR questionnaire was used for evaluation, which was composed of degrees of family fitness, cooperation, growth, affection and intimacy, with a score range of 0 to 10 . A total score 7 to 10,4 to 6 , and 0 to 3 represented a good family function, moderate dysfunction and a severe dysfunction, respectively [18]. (2) Evaluation of degree of nervous impairment: the National Institute of Health Stroke Scale (HINSS) was used for evaluation at the beginning of onset and at day 14 after onset. Each dimension score was divided into 3-5 levels with a range from 0 to 42 , and a higher score represented a more severe nervous impairments. (3) Evaluation of depression: evaluation was carried out at day 14 after disease onset. SDS was used for depression screening, which was a short- 


\section{Cellular Physiology Cell Physiol Biochem 2017;41:1393-1402

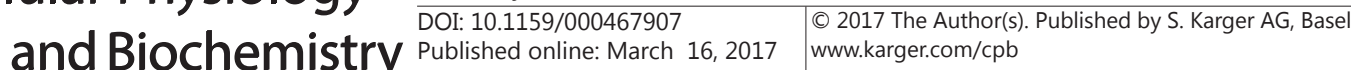

Zhang/Sui: Proton Magnetic Resonance Spectroscopy Study in PSD

term self-assessment scale, easy to use, and not affected by factors as age, gender and economic condition; therefore it is applied widely and especially suitable for the early identification of depression in general hospital. The assessment of the severity of depression preferred the HAMD scale. The HAMD scale was applied widely and in good reliability. It mainly evaluated the degree of the following items of the subjects: sense of guilty, suicidal ideation, sleep disorder, agitation or delay, somatic symptoms, weight loss, cognitive impairment, etc. (4) Assessment of leukodystrophy: evaluation was performed at day 14 after disease onset. The age related white matter change (ARWMC) scale was one of the most widely used scales in evaluating the location and severity of leukodystrophy, meanwhile, combined with the revision by Xiong,etc, it could be operated more easily $[19,20]$. Each cerebral hemisphere was divided into 5 areas, namely, frontal lobe, occipital lobe, temporal lobe, infratentorial area (including the cerebellum and brain stem) and basal ganglia region. Each area was scored individually ( 0 to 3 scores, 4 levels), and the final score was the sum of the scores for each area of bilateral cerebral hemispheres ( 0 to 30 scores).

Cranial MRI morphological information collection. The cranial morphological information was collected using 3.0T MRI with general sequences such as T1WI, T2WI and DWI at day 14 after disease attack. The infarction lesion was measured with the semi-automatic method. That is, DWI sequence was used to confirm responsible foci, and the profile of infarction lesion was delineated manually on each layer of DWI sequence, followed by calculating the area, multiplying the interlayer spacing and adding up all the infarcted levels to get the volume of infarction lesion. At last, the volume of infarction lesion was standardized using the following formula: standardized infarction lesion volume $=$ infarction lesion volume $\times$ mean cranial median sagittal plane area/the median sagittal plane area of patient, and PACS system was adopted for measurement and calculation [21]. Scanning parameters were listed below: for axial T1WI RTSE, TR=2000ms, TE=20ms, IR=800ms, layer thickness $6 \mathrm{~mm}$, spacing $1 \mathrm{~mm}, \mathrm{NSA}=1$, matrix $=400 \times 351$ and TSE factor=7; for T2WI DRIVE, $\mathrm{TR}=3000 \mathrm{~ms}$, TE $=80 \mathrm{~ms}$, layer thickness $6 \mathrm{~mm}$, spacing $1 \mathrm{~mm}$, NSA=1, matrix $=484 \times 377$ and TSE factor $=15$; for FLAIR sequence, $T R=11000 \mathrm{~ms}, T E=120 \mathrm{~ms}, I R=2800 \mathrm{~ms}$, layer thickness $6 \mathrm{~mm}$, spacing $1 \mathrm{~mm}, \mathrm{NSA}=1$, matrix $=240 \times 187$, TSE factor=32; for sagittal T2WI TSE, TR=2123ms, TE $=80 \mathrm{~ms}$, layer thickness $5 \mathrm{~mm}$, spacing $1 \mathrm{~mm}$, NSA=1, matrix=384×336 and TSE factor=19.

Cerebellar metabolic change observed by the hydrogen proton magnetic resonance spectroscopy $\left({ }^{1} H-M R S\right)$. Scanning was performed at day 14 after disease attack. Routine MRI with TSE sequence was used to exclude the brain lesions. Bilateral medipeduncle were chosen symmetrically on routine MRI T1WI or T2WI images as spectrum region of interests (ROI). Voxel size was $1 \mathrm{~cm} \times 1 \mathrm{~cm} \times 1.5 \mathrm{~cm}$, and the structures such as skull, adipose, air cavity and cerebrospinal fluid were kept away from. The saturated zone was adopted to eliminate the effect of peripheral tissues on scan results. MRS scan was performed using MRSspecified cranial coil with the Point-Resolved Echo Spin Spectroscopy (PRESS) sequence in the PROBE-SV software package. Curve-fitting, zero padding and Fourier transformation and adjustment of the phase and the baseline were performed using random LEONARDO software automatically. Scan parameters showed $\mathrm{TR}=1500 \mathrm{~ms}$ and $\mathrm{TE}=35 \mathrm{~ms}$. Transmission and reception of gain adjustment shimming and anhydrous inhibition scanning were completed automatically. The full width at half maximum of water peak was less than 3-5Hz. By observing metabolite changes, we found that ${ }^{1} \mathrm{H}-\mathrm{MRS}$ of normal brain tissue on different TE showed different types of metabolite, and long TE was chosen in this study. So the measured metabolites were NAA, Cho and Cr, with the corresponding chemical shift was $2.02 \mathrm{ppm}, 3.22 \mathrm{ppm}$, and 3.02ppm, respectively. The relative concentration change of NAA and Cho metabolite was observed, and the ratio of NAA/Cr, Cho/ $\mathrm{Cr}$ and Cho/NAA was calculated. As an energetic metabolite, the concentration of $\mathrm{Cr}$ in brain metabolite was the most stable and its quantization was relatively constant under different conditions including the pathological conditions, therefore, it was usually chosen as the reference substance to reflect the change of other metabolites. The relative concentration ratio of NAA/Cr to Cho/Cr could reflect the change of NAA and Cho more objectively. All scanning was done by the same professional doctor of rich experience, recorded in light disk and measured at the same time.

\section{Statistical analysis}

Data were expressed as mean \pm standard deviation $(\bar{x} \pm s)$. SPSS 17.0 Software was adopted for statistical analysis and treatment. Chi-square analysis was used for quantitative data, and one-way ANOVA was employed for qualitative data. $t$ test was used for comparison of means obtained from the two sample. Multiple linear regression analysis was adopted for the factors showing a statistical significance in the oneway ANOVA test. $P<0.05$ was considered statistically significant, and all tests were two-sided. 


\section{Results}

\section{Clinical data comparison}

The difference of age, gender, education level, occupations, economic conditions, health insurance, past medical history (hypertension, diabetes, hyperlipidemia and cardiac disease), family history (hypertension, cardiac disease and stroke) and previous life style between groups were of no statistical significance $(P>0.05)$. The difference of NIHSS scores between PSD group and CONT group at the early onset was of no statistical significance $(P>$ $0.05)$; while the difference was significant at day 14 after disease onset $(P<0.05)$. The family APGAR score, HAMD score and SDS score in PSD group was significantly different from those in CONT group and NORM group $(P<0.05)$. See Table 1 .

\section{Comparison of imaging data}

Comparison of leukodystrophy and infarct volume. There was no significant difference in the leukodystrophy ARWMC scores of frontal lobe, occipital lobe, temporal lobe, infratentorial area, and basal ganglia region between the groups $(P>0.05)$; while the total

Table 1. The clinical data of the PSD, CONT and NORM group. Note: ${ }^{*}$ Compare PSD group with CONT group and NORM group through SNK test, $P<0.05$; compare CONT group with NORM group, $P>0.05 ;{ }^{* *} P<0.05$

\begin{tabular}{lcccc}
\hline Indicators & PSD group & CONT group & NORM group & $P$ value \\
\hline Number of subjects & 20 & 20 & 20 & - \\
Age (years) & $54.8 \pm 9.9$ & $55.7 \pm 7.8$ & $53.0 \pm 10.5$ & 0.646 \\
Gender (M/F) & $11 / 9$ & $12 / 8$ & $10 / 10$ & 0.817 \\
Education length & $9.4 \pm 3.0$ & $8.9 \pm 2.6$ & $9.5 \pm 2.6$ & 0.770 \\
Physical work (Number (\%)) & $11(55.0)$ & $9(45.0)$ & $7(35.0)$ & 0.446 \\
Per capita income $>$ 1000 yuan (Number (\%)) & $13(65.0)$ & $12(60.0)$ & $13(65.0)$ & 0.931 \\
Enjoy health insurance (Number (\%)) & $12(60.0)$ & $10(50.0)$ & $13(65.0)$ & 0.619 \\
History of hypertension (Number (\%)) & $11(55.0)$ & $13(65.0)$ & $10(50.0)$ & 0.622 \\
History of diabetes (Number (\%)) & $7(35.0)$ & $6(30.0)$ & $9(45.0)$ & 0.605 \\
History of cardiac disease (Number (\%)) & $5(25.0)$ & $6(30.0)$ & $3(15.0)$ & 0.521 \\
History of hyperlipidemia (Number (\%)) & $8(40.0)$ & $10(50.0)$ & $8(40.0)$ & 0.762 \\
Family history of hypertension (Number (\%)) & $8(40.0)$ & $7(35.0)$ & $5(25.0)$ & 0.592 \\
Family history of cardiac disease (Number (\%)) & $9(45.0)$ & $6(30.0)$ & $7(35.0)$ & 0.605 \\
Family history of stroke (Number (\%)) & $7(35.0)$ & $9(45.0)$ & $10(50.0)$ & 0.622 \\
Previous lifestyle & $1.95 \pm 1.10$ & $1.70 \pm 1.17$ & $1.75 \pm 1.06$ & 0.756 \\
Family APGAR scale scores & $6.75 \pm 1.97$ & $7.50 \pm 1.40$ & $8.00 \pm 1.26$ & $0.048^{*}$ \\
NIHSS scores at early onset & $6.95 \pm 2.26$ & $6.25 \pm 2.29$ & - & 0.332 \\
NIHSS scores at day 14 & $5.65 \pm 1.84$ & $3.00 \pm 1.17$ & - & $0.030^{* *}$ \\
HAMD scores & $13.65 \pm 3.86$ & $2.65 \pm 1.90$ & $2.55 \pm 1.85$ & $0.000^{*}$ \\
SDS standard scores & $57.95 \pm 4.14$ & $29.10 \pm 5.80$ & $26.80 \pm 3.90$ & $0.000^{*}$ \\
\hline
\end{tabular}

Table 2. The comparison of leukodystrophy and infarct volume of the PSD, CONT and NORM group. Note: * Compare PSD group with CONT group and NORM group through SNK test, $P<0.05$; Compare CONT group with NORM group, $P>0.05$

\begin{tabular}{lcccc}
\hline \multicolumn{1}{c}{ Indicators } & PSD group & CONT group & NORM group & $P$ value \\
\hline ARWMC scores & & & & \\
Frontal lobe & $1.50 \pm 1.19$ & $1.55 \pm 1.23$ & $1.20 \pm 1.06$ & 0.592 \\
Occipital lobe & $1.84 \pm 1.42$ & $1.55 \pm 1.28$ & $1.39 \pm 1.19$ & 0.541 \\
Temporal lobe & $0.65 \pm 0.74$ & $0.60 \pm 0.75$ & $0.52 \pm 0.61$ & 0.792 \\
Infratentorial region & $0.70 \pm 0.66$ & $0.55 \pm 0.60$ & $0.43 \pm 0.51$ & 0.413 \\
Basal ganglion region & $1.29 \pm 1.17$ & $1.10 \pm 0.97$ & $0.75 \pm 0.79$ & 0.214 \\
Total scores & $5.95 \pm 2.11$ & $5.20 \pm 2.26$ & $4.25 \pm 1.89$ & $0.044^{*}$ \\
Lesion was at the left side (Number(\%)) & $11(55.0)$ & $8(40.0)$ & - & 0.342 \\
Standardized infarct volume (ml) & $4.33 \pm 1.80$ & $4.19 \pm 2.12$ & - & 0.697 \\
\hline
\end{tabular}


Table 3. The comparison of metabolite indicators tested by MRS in PSD, CONT and NORM group. Note: The ipsilateral lesion in NORM group referred the left side, while the contralateral lesion was the left side; $\mathrm{P}_{1}$ for intra-group comparison of bilateral cerebellum. ${ }^{*} P<0.05$, ** Comparing PSD group with CONT group and NORM group through SNK test, $P<0.05$, respectively; Comparing CONT group with NORM group, $P>0.05$

\begin{tabular}{lccccccccc}
\hline & Ipslateral & $\begin{array}{l}\mathrm{NAA} / \mathrm{Cr} \\
\text { Contralateral }\end{array}$ & $\begin{array}{c}\mathrm{P}_{1} \\
\text { value }\end{array}$ & Ipslateral & $\begin{array}{l}\mathrm{Cho} / \mathrm{Cr} \\
\text { Contralateral }\end{array}$ & $\begin{array}{l}P_{1} \\
\text { value }\end{array}$ & $\begin{array}{c}\text { Cho/NA } \\
\text { Ipslateral }\end{array}$ & $\begin{array}{c}P_{1} \\
\text { Contralateral }\end{array}$ \\
\hline $\begin{array}{l}\text { PSD group } \\
\text { CONT }\end{array}$ & $1.044 \pm 0.053$ & $1.038 \pm 0.038$ & 0.695 & $0.779 \pm 0.054$ & $0.897 \pm 0.087$ & $0.000^{*}$ & $0.748 \pm 0.063$ & $0.864 \pm 0.082$ & $0.000^{*}$ \\
group & $1.048 \pm 0.054$ & $1.047 \pm 0.041$ & 0.932 & $0.750 \pm 0.065$ & $0.776 \pm 0.059$ & 0.074 & $0.719 \pm 0.084$ & $0.743 \pm 0.069$ & 0.178 \\
NORM & $1.052 \pm 0.045$ & $1.056 \pm 0.053$ & 0.806 & $0.761 \pm 0.086$ & $0.771 \pm 0.090$ & 0.529 & $0.725 \pm 0.096$ & $0.729 \pm 0.093$ & 0.791 \\
group & 0.145 & 0.801 & - & 0.863 & 16.047 & - & 0.713 & 16.459 & - \\
$\begin{array}{l}\text { F value } \\
\text { P value }\end{array}$ & 0.865 & 0.454 & - & 0.421 & $0.000^{* *}$ & - & 0.495 & $0.000^{* *}$ & - \\
\hline
\end{tabular}

scores of different regions were significant different between the three group $(P<0.05)$. There was no statistical significance of the difference in the side of responsible focus and standardized infarct volume between PSD group and CONT group $(P>0.05)$. See Table 2.

Comparison of metabolite indicators tested by MRS. This study compared the ipsilateral and contralateral cerebellum of the ischemic cerebral stroke lesion in PSD group and CONT group with the NORM group, respectively. No significant difference was noted in the left and right cerebellar NAA/ $\mathrm{Cr}, \mathrm{Cho} / \mathrm{Cr}$ and Cho/NAA ratio $(P>0.05)$. The ipsilateral and contralateral cerebellar $\mathrm{NAA} / \mathrm{Cr}$, Cho/Cr and Cho/NAA ratio in CONT group was not significantly different from those in NORM group $(P>0.05)$. Compared with NORM group and CONT group, the difference of the ipsilateral and contralateral cerebellar NAA/Cr ratio in PSD group was not statistically significant $(P>0.05)$; while the contralateral cerebellar $\mathrm{Cho} / \mathrm{Cr}$ and Cho/NAA ratio was significantly higher $(P$ $<0.05$ ), and the ipsilateral cerebellar Cho/ $\mathrm{Cr}$ and Cho/NAA ratio was not significantly different $(P>0.05)$. Besides, the contralateral cerebellar Cho/Cr and Cho/NAA ratio was statistically higher than that of the ipsilateral values $(P<0.05)$. See Table 3 and Figure 1-4.

Multiple linear regression analysis of depression-related influence factors at the acute phase of ischemic stroke. The independent variables of the multiple linear regression model obtained from the results

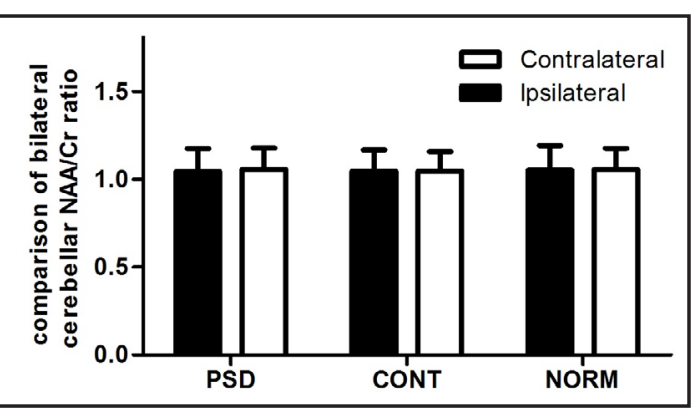

Fig. 1. Intragroup and intergroup comparison of bilateral cerebellar NAA/Cr ratio.

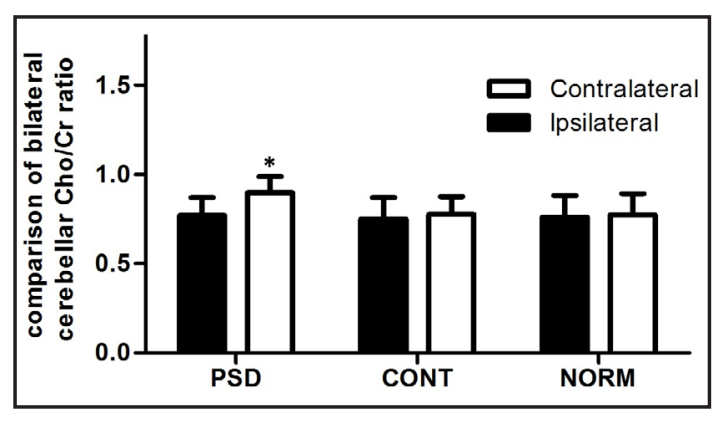

Fig. 2. Intragroup and intergroup comparison of bilateral cerebellar Cho/Cr ratio. $\left({ }^{*} \mathrm{P}<0.05\right)$.

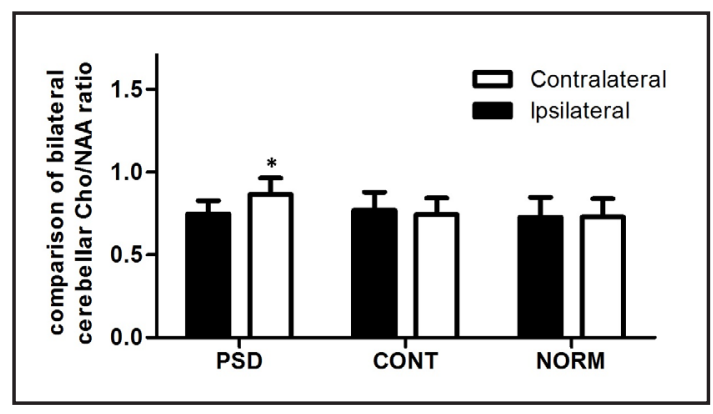

Fig. 3. Intragroup and intergroup comparison of bilateral cerebellar Cho/NAA ratio. $\left({ }^{*} \mathrm{P}<0.05\right)$. above included family APGAR questionnaire scores, ARWMC scores, NIHSS scores at day 14 after attack, the ratio of Cho/Cr of the contralateral cerebellum to Cho/NAA, and the dependent variable was HAMD scores. The multiple linear regression analysis showed that the ratio of $\mathrm{Cho} / \mathrm{Cr}$ of the contralateral 
Fig. 4. A. The ipsilateral cerebellum MRS image of the lesion in PSD patients; B. The contralateral cerebellum MRS image of the lesion in PSD patients.
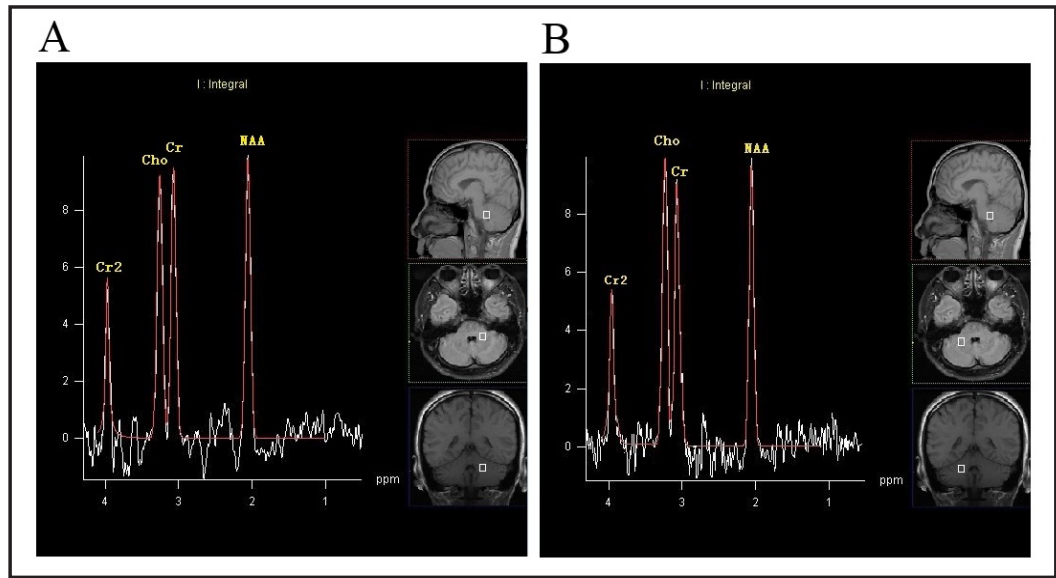

Table 4. Multiple linear regression analysis of depression-related influence factors at the acute phase of ischemic stroke. Note: ${ }^{*}<0.05$

\begin{tabular}{|c|c|c|c|c|c|c|c|}
\hline \multirow[t]{2}{*}{ Indicators } & \multicolumn{2}{|c|}{ Non-standardized } & \multirow[t]{2}{*}{$\begin{array}{l}\text { Standardizedpartial } \\
\text { regression coefficient }\end{array}$} & \multirow{2}{*}{$\begin{array}{c}t \\
\text { value }\end{array}$} & \multirow{2}{*}{$\begin{array}{c}P \\
\text { value }\end{array}$} & \multicolumn{2}{|c|}{$\begin{array}{l}95 \% \text { confidence } \\
\text { intervals }\end{array}$} \\
\hline & $\begin{array}{c}\text { Partial } \\
\text { regression } \\
\text { coefficient }\end{array}$ & $\begin{array}{l}\text { Standard } \\
\text { error }\end{array}$ & & & & $\begin{array}{l}\text { Lower } \\
\text { limit }\end{array}$ & $\begin{array}{l}\text { Upper } \\
\text { limit }\end{array}$ \\
\hline APGAR score & -0.283 & 0.459 & -0.145 & $\begin{array}{c}- \\
0.617\end{array}$ & 0.546 & -1.261 & 0.694 \\
\hline $\begin{array}{l}\text { NIHSS score } \\
\text { at day } 14\end{array}$ & -0.611 & 0.489 & -0.292 & $\begin{array}{c}- \\
1.250\end{array}$ & 0.231 & -1.653 & 0.431 \\
\hline $\begin{array}{l}\text { Total ARWMC } \\
\text { score }\end{array}$ & -0.142 & 0.417 & -0.078 & 0.341 & 0.738 & -1.031 & 0.747 \\
\hline Cho/Cr ratio & 22.151 & 9.973 & 0.498 & 2.221 & $0.042^{*}$ & 0.893 & 43.409 \\
\hline
\end{tabular}

Table 5. Multiple linear regression analysis of depression-related influence factors at the acute phase of ischemic stroke. Note:* $P<0.05$

\begin{tabular}{|c|c|c|c|c|c|c|c|}
\hline \multirow[t]{2}{*}{ Indicators } & \multicolumn{2}{|c|}{ Non-standardized } & \multirow[t]{2}{*}{$\begin{array}{l}\text { Standardizedpartial } \\
\text { regression coefficient }\end{array}$} & \multirow[t]{2}{*}{$\begin{array}{c}t \\
\text { value }\end{array}$} & \multirow[t]{2}{*}{$\begin{array}{c}P \\
\text { value }\end{array}$} & \multicolumn{2}{|c|}{$\begin{array}{l}95 \% \text { confidence } \\
\text { intervals }\end{array}$} \\
\hline & $\begin{array}{l}\text { regression } \\
\text { coefficient }\end{array}$ & error & & & & & \\
\hline APGAR score & -0.337 & 0.445 & -0.172 & $\begin{array}{c}- \\
0.756\end{array}$ & 0.461 & -1.286 & 0.612 \\
\hline $\begin{array}{l}\text { NIHSS score } \\
\text { at day } 14\end{array}$ & -0.651 & 0.473 & -0.311 & $\begin{array}{c}- \\
1.376\end{array}$ & 0.189 & -1.659 & 0.357 \\
\hline $\begin{array}{l}\text { Total ARWMC } \\
\text { scores }\end{array}$ & -0.176 & 0.398 & -0.097 & $\overline{-}-\overline{442}$ & 0.665 & -1.026 & 0.673 \\
\hline $\begin{array}{l}\text { Cho/NAA } \\
\text { ratio }\end{array}$ & 25.926 & 10.235 & 0.548 & 2.533 & $0.023^{*}$ & 4.109 & 47.742 \\
\hline
\end{tabular}

cerebellum was positively related with the HAMD scores $\left(\mathrm{R}=0.555, \mathrm{R}^{2}=0.308, \mathrm{P}<0.05\right)$, while the ratio of Cho/NAA of the contralateral cerebellum was positively related with HAMD scores $\left(\mathrm{R}=0.597, \mathrm{R}^{2}=0.356, \mathrm{P}<0.05\right)$. See the Table 4 and Table 5.

\section{Discussion}

At present, the pathogenesis of PSD is still unclear. Our previous basic studies have proved a significant alteration of the cerebellar structure and function in PSD rat $[22,23]$. The MRS technique has been proved to be sensitive in detecting the metabolic change of cerebellum when the structure is intact [24]. The present study is to evaluate whether the PSD patients' cerebellar function (metabolism) change through ${ }^{1} \mathrm{H}-\mathrm{MRS}$ technique in clinic.

According to our research results, the contralateral cerebellar $\mathrm{Cho} / \mathrm{Cr}$ ratio of the lesion in PSD group was significantly higher than that of the control group, which confirmed the 
alteration of cerebellar metabolism in PSD patients. As one of the components of phospholipid metabolism of cell membrane, Cho can reflect the transporting function of cell membrane and the proliferation state of cells, and is the precursor of acetyl choline, a neurotransmitter which is closely related to emotion, and is the pathophysiological foundation of emotional disturbance. In the present study, the ratio of $\mathrm{Cho} / \mathrm{Cr}$ was elevated, which suggested that PSD might be related to accelerated phospholipid turnover of cerebellar neural cell membrane and alteration of membrane structure. There is rare report of MRS study about the relation between cerebellum and PSD worldwide. With regard to infantile autism or other psychiatric disorders, some reports showed the cerebellar Cho concentration was significantly elevated compared with the control group [12, 25], which was similar to ours.

Our study also measured the ratio of cerebellar NAA/Cr, and the results showed no significant difference between PSD group and control group. As NAA is associated with the integrity of neuron and reflects the function state of neuron, it is an ideal marker of undeveloped nerve tissues. From this reason, we concluded that the pathogenesis of acute PSD might be non-neuronal alteration. As shown by An et al., no abnormal alteration of cerebellar NAA concentration was observed in PSD patients [26]. Meanwhile, we measured the bilateral cerebellar Cho/NAA ratio, and the results indicated the contralateral ratio of PSD group was elevated, while the ipsilateral ratio was not statistically different. This indicated that the change of cerebellar Cho relative to NAA level was more significant in PSD patients. Interestingly, we also found that the bilateral cerebellar Cho/Cr, NAA/Cr and Cho/NAA in CONT group were not different from those in NORM group, revealing no metabolic change in the patients without depression after stroke. This further supported our hypothesis, that is, PSD was related to the alteration of cerebellar metabolism.

There is rare report of the cerebellar functional (metabolic) alteration in PSD patients. But in the field of depression, many studies also found the change of cerebellar function in depression patients through functional MRI (fMRI) technique. Besides, many studies have displayed that the cerebellar structure varies, which necessarily leads to the functional changes [11, 27, 28]. Lassalle-Lagadec et al. found significantly decreased volume of PSD patients' cerebellum [28]. Moreover, the magnetic resonance diffusion tensor imaging study about depression also showed obvious abnormality of patients' cerebellar white matter network. Namely, the cerebellar structure has changed [11].

Besides, we found that in PSD patients, only the contralateral cerebellar $\mathrm{Cho} / \mathrm{Cr}$ ratio of the lesion changed while the ipsilateral ratio was not different from that in the control group. According to evidence from massive experiments in recent years, crossed cerebellar diaschisis (CCD) (i.e., discontinuation of cortex-cerebellopontine (CPC) pathway caused by various brain injuries) may cause that cortex irritability impulse cannot transmit to the contralateral cerebellum through pons and medipeduncle normally, thus leading to a decrease of blood flow and metabolism of contralateral cerebellum and further inhibiting the function [29]. CPC pathway is composed of the fiber bundles from cortex to the ipsilateral pons and from pons to the contralateral cerebellum [30]. As shown in the study of Strakowski et al., the cortex-subcortex reticular system and limbic system were the brain function center of the affective disorder, and their functional abnormality might be origin of affective disorder [31]. Cerebellum has wide fibrous connections with such regions, among which the most important connection is the CPC pathway [25]. We speculated that the lesions at the basal ganglion regions of PSD patients in our study caused the discontinuation of such pathway to induce CCD, affecting the contralateral cerebellar metabolism and further leading to PSD.

Our study preliminarily proved changes of cerebellar metabolism in PSD patients. Many researches have confirmed that electric stimulation could improve the symptoms of PSD patients [27, 32]. In summary, we speculated that the alteration of cerebellum caused PSD. However, the degree of family function satisfaction, severity of leukodystrophy and neuro function defect at day 14 after disease onset were also shown to be associated with PSD. By further multiple linear regression analysis, a positive correlation was noted between the contralateral cerebellar Cho/Cr with Cho/NAA ratio and HAMD score, but not between the degree of family function satisfaction, severity of leukodystrophy and neuro function defect

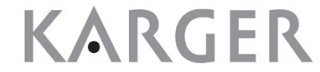




\section{Cellular Physiology Cell Physiol Biochem 2017;41:1393-1402

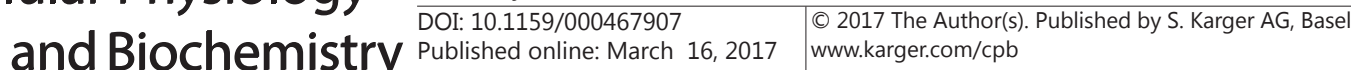 \\ Zhang/Sui: Proton Magnetic Resonance Spectroscopy Study in PSD}

at day 14 after disease onset and HAMD score. Based on the present study, the degree of cerebellar metabolism variation was correlated with the severity of PSD. Similar studies around the world supported this conclusion, for example, in DTI image study by Peng et al., the abnormality of cerebellar white matter network in refractory depression patients showed a significant correlation with the severity of depression [11]. While Sui et al. also indicated the degree of cerebellar volume decrease was associated with the severity of PSD [23].

Since the present study had small sample size, and other metabolites during PSD (small molecules such as inositol, glutamic acid) were not determined, some useful information might be missed. Moreover, even though we collected and excluded many PSD-related factors, some unknown factors might still exist.

In summary, the cerebellar metabolism of PSD patients changes after the disease onset (i.e., the cerebellar injury occurred after stroke), and the severity of injury is related to the severity of PSD. The present study preliminarily confirms that cerebellum is involved in the development of PSD, providing a new insight for investigating the pathogenesis of PSD and a new clue for its early prevention.

\section{Acknowledgement}

The study was supported by National Natural Science Foundation of China (Grant Nos. 81241050 and 81371461), Natural Science Foundation of Liaoning Province (Grant No. 2013022018), and Liaoning Medical College Youth Foundation (Grant No. Y2012Z014).

\section{Disclosure Statement}

Authors claim no conflict of interest.

\section{References}

1 Hackett ML, Kohler S, O'Brien JT, Mead GE: Neuropsychiatric outcomes of stroke. Lancet Neurol 2014;13:525-534.

2 Yuan HW, Wang CX, Zhang N, Bai Y, Shi YZ, Zhou Y, Wang YL, Zhang T, Zhou J, Yu X, Sun XY, Liu ZR, Zhao XQ Wang YJ: Poststroke depression and risk of recurrent stroke at 1 year in a Chinese cohort study. PLoS One 2012; 7:e46906.

3 Provinciali L, Coccia M: Post-stroke and vascular depression: a critical review. Neurol Sci 2002;22:417-428.

4 Hackett ML, Anderson CS, House A, Xia J: Interventions for treating depression after stroke. Cochrane Database Syst Rev 2008;10.1002/14651858.CD003437.pub3CD003437.

5 Altieri M, Maestrini I, Mercurio A, Troisi P, Sgarlata E, Rea V, Di Piero V, Lenzi GL: Depression after minor stroke: prevalence and predictors. Eur J Neurol 2012;19:517-521.

6 Bhogal SK, Teasell R, Foley N, Speechley M: Lesion location and poststroke depression: systematic review of the methodological limitations in the literature. Stroke 2004;35:794-802.

-7 Eccles JC, Llinas R, Sasaki K: Inhibitory systems in the cerebellar cortex. Proc Aust Assoc Neurol 1965;3:714.

8 Eccles JC, Llinas R, Sasaki K: The excitatory synaptic action of climbing fibres on the Purkinje cells of the cerebellum. J Physiol 1966;182:268-296.

-9 Chan-Palay V, Palay SL, Billings-Gagliardi SM: Meynert cells in the primate visual cortex. J Neurocytol 1974;3:631-658.

10 Kumar A, Bhoi SK, Kalita J, Misra UK: Central Post Stroke Pain Can Occur with Normal Sensation. Clin J Pain 2015;10.1097/AJP.0000000000000344

11 Peng HJ, Zheng HR, Ning YP, Zhang Y, Shan BC, Zhang L, Yang HC, Liu J, Li ZX, Zhou JS, Zhang ZJ, Li LJ: Abnormalities of cortical-limbic-cerebellar white matter networks may contribute to treatment-resistant depression: a diffusion tensor imaging study. BMC Psychiatry 2013;13:72. 


\section{Cellular Physiology Cell Physiol Biochem 2017;41:1393-1402

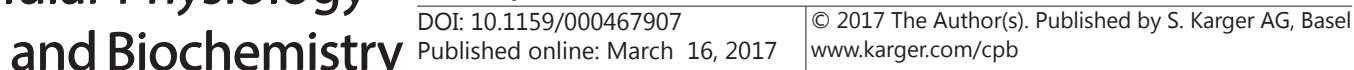

12 Gabis L, Wei H, Azizian A, DeVincent C, Tudorica A, Kesner-Baruch Y, Roche P, Pomeroy J: 1H-magnetic resonance spectroscopy markers of cognitive and language ability in clinical subtypes of autism spectrum disorders. J Child Neurol 2008;23:766-774.

13 Llinas R, Yarom Y: Electrophysiology of mammalian inferior olivary neurones in vitro. Different types of voltage-dependent ionic conductances. J Physiol 1981;315:549-567.

14 Kimura T, Sugimori M, Llinas RR: Purkinje cell long-term depression is prevented by T-588, a neuroprotective compound that reduces cytosolic calcium release from intracellular stores. Proc Natl Acad Sci U S A 2005;102:17160-17165.

-15 Mast BT, MacNeill SE, Lichtenberg PA: Post-stroke and clinically-defined vascular depression in geriatric rehabilitation patients. Am J Geriatr Psychiatry 2004;12:84-92.

-16 Tsai TH, Lu CH, Wallace CG, Chang WN, Chen SF, Huang CR, Tsai NW, Lan MY, Sung PH, Liu CF, Yip HK: Erratum to: Erythropoietin improves long-term neurological outcome in acute ischemic stroke patients: a randomized, prospective, placebo-controlled clinical trial. Crit Care 2016;20:78.

17 Taylor-Piliae RE, Hoke TM, Hepworth JT, Latt LD, Najafi B, Coull BM: Effect of Tai Chi on physical function, fall rates and quality of life among older stroke survivors. Arch Phys Med Rehabil 2014;95:816-824.

18 Hackett ML, Anderson CS, House AO: Interventions for treating depression after stroke. Cochrane Database Syst Rev 2004;10.1002/14651858.CD003437.pub2CD003437.

19 Wahlund LO, Barkhof F, Fazekas F, Bronge L, Augustin M, Sjogren M, Wallin A, Ader H, Leys D, Pantoni L, Pasquier F, Erkinjuntti T, Scheltens P: A new rating scale for age-related white matter changes applicable to MRI and CT. Stroke 2001;32:1318-1322.

20 Xiong Y, Yang J, Wong A, Wong CH, Chan SS, Li HH, Tam LH, Bao JW, Wong GC, Chen X, Chu WC, Lee WK, Wong KS, Mok VC: Operational definitions improve reliability of the age-related white matter changes scale. Eur J Neurol 2011;18:744-749.

-21 Ferguson KJ, Wardlaw JM, Edmond CL, Deary IJ, Maclullich AM: Intracranial area: a validated method for estimating intracranial volume. J Neuroimaging 2005;15:76-78.

-22 Iacoboni M, Padovani A, Di Piero V, Lenzi GL: Post-stroke depression: relationships with morphological damage and cognition over time. Ital J Neurol Sci 1995;16:209-216.

-23 Sui R, Zhang L, Min L, Yuan J, Li X: Cerebellar dysfunction may play an important role in post-stroke depression. Med Hypotheses 2009;72:643-646.

-24 Wilkinson ID, Hadjivassiliou M, Dickson JM, Wallis L, Grunewald RA, Coley SC, Widjaja E, Griffiths PD: Cerebellar abnormalities on proton MR spectroscopy in gluten ataxia. J Neurol Neurosurg Psychiatry 2005;76:1011-1013.

25 Stanley JA: In vivo magnetic resonance spectroscopy and its application to neuropsychiatric disorders. Can J Psychiatry 2002;47:315-326.

26 An D, Dubeau F, Gotman J: BOLD responses related to focal spikes and widespread bilateral synchronous discharges generated in the frontal lobe. Epilepsia 2015;56:366-374.

-27 Wang J, Dong WW, Zhang WH, Zheng J, Wang X: Electrical stimulation of cerebellar fastigial nucleus: mechanism of neuroprotection and prospects for clinical application against cerebral ischemia. CNS Neurosci Ther 2014;20:710-716.

-28 Lassalle-Lagadec S, Catheline G, Mayo W, Dilharreguy B, Renou P, Fleury O, Allard M, Swendsen J, Sibon I: Cerebellum involvement in post-stroke mood: a combined ecological and MRI study. Psychiatry Res 2013;212:158-160.

29 Rubin G, Levy EI, Scarrow AM, Firlik AD, Karakus A, Wechsler L, Jungreis CA, Yonas H: Remote effects of acute ischemic stroke: A xenon CT cerebral blood flow study. Cerebrovasc Dis 2000;10:221-228.

30 Kim J, Lee SK, Lee JD, Kim YW, Kim DI: Decreased fractional anisotropy of middle cerebellar peduncle in crossed cerebellar diaschisis: diffusion-tensor imaging-positron-emission tomography correlation study. AJNR Am J Neuroradiol 2005;26:2224-2228.

-31 Strakowski SM, Delbello MP, Adler CM: The functional neuroanatomy of bipolar disorder: a review of neuroimaging findings. Mol Psychiatry 2005;10:105-116.

-32 Stein C, Fritsch CG, Robinson C, Sbruzzi G, Plentz RD: Effects of Electrical Stimulation in Spastic Muscles After Stroke: Systematic Review and Meta-Analysis of Randomized Controlled Trials. Stroke 2015;46:21972205. 